\title{
TYPED FEATURE STRUCTURES AS DESCRIPTIONS
}

\author{
Paul John King*
}

\section{Seminar für Sprachwissenschaft, Eberhard-Karls-Universität ${ }^{\dagger}$}

\begin{abstract}
A description is an entity that can be interpreted as true or false of an object, and using feature structures as descriptions accrues several computational benefits. In this paper, I create an explicit interpretation of a typed feature structure used as a description, define the notion of a satisfiable feature structure, and create a simple and eflective algorithm to decide if a feature structure is satisfiable.
\end{abstract}

\section{INTRODUCTION}

Describing objects is one of several purposes for which linguists use feature structures. $\Lambda$ description is an entity that can be interpreted as true or false of an object. For example, the conventional interpretation of the description 'it is black' is true of a soot prarticle, but false of a snowflake. Therefore, any use of a feature structure to describe an object demands that the fcature structure can be interpreted as true or false of the object. In this paper, I tailor the semantics of [KING 1989] to suit the typed feature structures of [CARPLN'TER 1992], and so create an explicit interpretation of a typed feature structure used as a description. I then use this interpretation to define the notion of a satisfable feature structure.

Though no feature structure algebra provides descriptions as expressive as those provided by a feature logic, using feature structures to describe objects profits from a large stock of available computational techniques to represent, test and process featurc structures, In this paper, I demonstrate the computational benefits of marrying a tractable syntiax and an explicit semantics by creating a simple and effective algorithm to decide the satisfiability

\footnotetext{
*The research presented in this paper was sponsored by Teilprojekt $\mathrm{B4}$ "Comstraints on Grammar for Efficient Generation" of the Sonderforschungsbereich 340 of the Deutsche Forschungsgemeinschaft. I also wish to thank Bob Carpenter, Dale Gerdemann, Thilo Götz and Jemnifer King for their invaluable help with this paper.

tWilhelmstr. 113, 72074 Tülsingen, Germany.
} binail: kingosfs.nphil.uni-tuebingen.de. of a feature structure. Gerdemann and Götz's Troll type resolution system implements both the semantics and an efficient refinement of the satisfiability algorithm I present here (see [GöT\% 1993], [GeRdimann AND King 1994] and [GERDEMANN (FC)]).

\section{A FEATURE STRUCTURE SEMANTICS}

A signature provides the symbols from which to construct typed feature structures, and an interpretation gives those symbols meaning.

Definition 1. $\Sigma$ is a signature iff

$\Sigma$ is a sextuple $\langle\mathfrak{Q}, \mathfrak{T}, \preceq, \mathfrak{S}, \mathfrak{A}, \mathfrak{F}\rangle$,

$\mathbb{Q}$ is a set,

$\langle\mathfrak{T}, \preceq\rangle$ is a partial order,

$\mathfrak{S}=\left\{\sigma \in \mathfrak{T} \mid \begin{array}{l}\text { for cach } \tau \in \mathfrak{T}, \\ \text { if } \sigma \leq \tau \text { then } \sigma=\tau\end{array}\right\}$,

2t is a set,

$\mathfrak{F}$ is a partial function from the Cartesian prodict of $\mathfrak{T}$ and $\mathfrak{A}$ to $\mathfrak{T}$, and

for each $\tau \in \mathfrak{T}$, each $\tau^{\prime} \in \mathfrak{T}$ and each $\alpha \in \mathfrak{A}$,

if $\mathfrak{F}(\tau, \alpha)$ is defined and $\tau \preceq \tau^{\prime}$

then $\mathfrak{F}\left(\tau^{\prime}, \alpha\right)$ is defined, and $\mathfrak{F}(\tau, \alpha) \preceq \mathfrak{F}\left(\tau^{\prime}, \alpha\right)$.

Jenceforth, I tacitly work with a signature $(\mathfrak{Q}, \mathfrak{T}, \preceq, \mathfrak{S}, \mathfrak{A}, \mathfrak{F}\rangle$. I call nembers of $\mathfrak{Q}$ states, nembers of $\tau$ types, $\preceq$ subsumption, members of $\mathfrak{G}$ species, members of $\mathfrak{A}$ attributes, and $\mathfrak{F}$ appropriateness.

Definition 2. $I$ is an interpretation iff

$I$ is a triple $\langle U, S, A\rangle$,

$U$ is a set,

$S$ is a total function from $U$ to $\mathfrak{S}$

$A$ is a total function from $\mathfrak{A}$ to the set of partial functions from $U$ to $U$,

for each $\alpha \in \mathfrak{A}$ and each $u \in U$, if $A(\alpha)(u)$ is defined

then $\mathfrak{F}(S(u), \alpha)$ is defined, and $\mathfrak{F}(S(u), \alpha) \preceq S(A(\alpha)(u))$, and

for each $\alpha \in \mathfrak{A}$ and each $u \in U$, if $\mathfrak{F}(S(u), \alpha)$ is defined

then $A(\alpha)(u)$ is defined.

Suppose that $I$ is an interpretation $(U, S, A)$. I call each member of $U$ an object in $I$. 
Each type denotes a set of objects in $I$. 'The denotations of the speecies partition $U$, and $S$ assigns each object, in $I$ the muique species whose denotation contains the object: object $u$ is in the denotation of species $\sigma$ iff $\sigma=S(u)$. Subsumption encodes a rolationship between the denotations of species and types: object $u$ is in the denotation of type $\tau$ if $\tau \preceq S(u)$. So, if $\tau_{1} \preceq \tau_{2}$ then the denotation of type $\tau_{1}$ contatins the denotation of type $\tau_{2}$.

Wach attribute denotes a partial function from the objects in $I$ to the objects in $I$, and $A$ assigns each attribute the partial function it denotes. Appropriateness encorles a relationship between the denotations of species and attributes: if $\mathfrak{F}\langle\sigma, x\rangle$ is defined then the denotation of attribute $\alpha$ acts upon each object in the denotation of species $\sigma$ to yield an object in the denotation of type $\mathfrak{F}\langle\sigma, \alpha\rangle$, but if $\mathfrak{F}\langle\sigma, \alpha\rangle$ is undefined then the denotation of athibute. $\alpha$ acts upon no object in the denotation of species $\sigma$. So, if $\tau\langle\tau, \sigma\rangle$ is clefined then the deuotation of attribute $\alpha$ acts tupon each olject in the denotation of type $\tau$ to yield an object. in the denotation of type $\mathfrak{F}(\tau, \sigma)$.

I call a finite sequence of attributes a pall, and write $\mathfrak{P}$ for the set of patis.

Dofinition 3. $P$ is the path interpretation function under $l$ ifl

$I$ is an interpretation $(U, S, A\rangle$,

$P$ is a tolal function from to the sed of partial functions from $U$ to $U$, and

for each $\left\langle\alpha_{1}, \ldots, \alpha_{n}\right) \in \mathfrak{P}$,

$l^{\prime}\left\langle\alpha_{1}, \ldots, \alpha_{n}\right\rangle$ is the functional composition of $A\left(\alpha_{1}\right), \ldots, A\left(\gamma_{n}\right)$.

I write $P_{I}$ for the path interpretation function under 1 .

Dofinition 4. $l$ is a foature structure iff $r^{\prime}$ is a qualruple $\langle Q, q, \delta, Q\rangle$,

$Q$ is a finite subset of $\mathfrak{Q}$,

$q \in Q$

$\delta$ is a finite partial function from the Cartesian product of $Q$ and 2 to $Q$,

$O$ is a total function from $Q$ to $T$, and

for each $q^{\prime} \in Q$,

for some $\pi \in \mathfrak{P}^{3}, \pi$ rums to $q^{\prime}$ in $w^{r}$, where $\left\langle\alpha_{1}, \ldots, \alpha_{n}\right\rangle$ rans to $\eta^{\prime}$ in $F^{\prime}$ ill

$\left\langle\alpha_{1}, \ldots, \alpha_{n}\right\rangle \in \mathfrak{P}$,

$q^{\prime} \in Q$, and

for some $\left\{q_{0}, \ldots, q_{n}\right\} \subseteq Q$,

$q=q_{0}$,

for each $i<n$,

$\delta\left(q_{i}, \alpha_{i+1}\right)$ is defined, and

$\delta\left(q_{i}, \alpha_{i+1}\right)=q_{i+1}$, and

$q_{n}=q^{\prime}$.

Finch feature structure is a connected Moore machine (see [MoorF, 1356]) with finitely many states, input alphabet $\mathfrak{A}$, and output alplabet $\mathfrak{T}$

Definition 5. $F$ is true of $u$ under $I$ iff

$F$ is a feabure structure $\langle Q, q, \delta, 0\rangle$,

$I$ is an interpretation $\langle U, S, A\rangle$,

$u$ is an object in $I$, and

for each $\pi_{1} \in \mathfrak{P}$, each $\pi_{2} \in \mathfrak{P}$ and each

$q^{\prime} \in Q$,

if $\pi_{1}$ runs to $q^{\prime}$ in $l^{\prime}$, and

$\pi:$ runs to $q^{\prime}$ in $l^{\prime}$

then $P_{I}\left(\pi_{1}\right)(u)$ is acfined, $P_{I}\left(\pi_{2}\right)(u)$ is defined, $P_{I}\left(\pi_{1}\right)(u)=P_{I}\left(\pi_{2}\right)(u)$, and $O\left(q^{\prime}\right) \preceq S\left(P_{I}\left(\pi_{\rfloor}\right)(u)\right)$.

Definition $6, l^{\prime}$ is a satisfiable feature structure ifl

$l^{\prime}$ is a leature structure, and

for some interpretation I and some object $u$ in $I, l$ is true of $u$ under $I$.

\section{MORPHS}

The ahundance of interpretations seens to prechude an effective algorithm to decide if a feature structure is satisfiable. However, I insert morphs between feature structures and objects to yield an interpretation frec characterisation of a satisfiable feature structure.

Dofinition 7. $M$ is a semi-morph iff

$M$ is a triple $\langle\Delta, \Gamma, \Lambda\rangle$,

$\Delta$ is a noncmply subset of $\mathfrak{P}$,

$I$ ' is an equivalence relation over $\Delta$,

for each $x \in \mathfrak{A}$, oach $\pi_{1} \in \mathfrak{P}$ and each $\pi_{2} \in \mathfrak{P}$,

if $\pi_{1} \alpha \in \Delta$ and $\left\langle\pi_{1}, \pi_{2}\right\rangle \in \mathrm{l}^{\prime}$

then $\left\langle\pi_{1} \alpha, \pi_{2} \gamma\right\rangle \in \mathrm{l}^{3}$,

$\Lambda$ is a total function from $\Delta$ to $\mathcal{S}$,

for each $\pi_{1} \in \mathfrak{P}$ and cach $\pi_{2} \in \mathfrak{P}$, if $\left\langle\pi_{1}, \pi_{2}\right\rangle \in$ I' then $\Lambda\left(\pi_{1}\right)=\Lambda\left(\pi_{2}\right)$, and

for each $\alpha \in \mathfrak{A}$ and cach $\pi \in \mathfrak{P}$,

if $\pi \alpha \in \Delta$

then $\pi \in \Delta, \mathfrak{F}(\Lambda(\pi), \alpha)$ is defined, and $\mathfrak{F}(\Lambda(\pi), \alpha) \preceq \Lambda(\pi \alpha)$.

Definition 8. $M$ is a morph ifl

$M$ is a semi-morph $(\Delta, l, \Lambda)$, and

for cach $\alpha \in \mathfrak{A}$ and cach $\pi \in \mathfrak{P}$,

if $\pi \in \Delta$ and $\mathfrak{F}(\Lambda(\pi), \alpha)$ is defined

then $\pi \alpha \in \Delta$.

Wach morph is the Moshier abstraction (see [Mosinm 1988]) of a connected and totally well-typed (see [CARPENTWR 1992]) Moore machine with possibly infinitely many states, input alphabet $\mathfrak{A}$, and output alphabet $\mathcal{S}$. 
Definition 9. $M$ abstracts $u$ under $I$ if

$M$ is a morph $\langle\triangle, \Gamma, \Lambda\rangle$,

$I$ is an interpretation $\langle U, S, A\rangle$,

$u$ is an object in $I$,

for cach $\pi_{1} \in \mathfrak{P}$ and each $\pi_{2} \in \mathfrak{P}$,

$\left\langle\pi_{1}, \pi_{2}\right\rangle \in \Gamma^{\prime}$

iff $P_{I}\left(\pi_{1}\right)(u)$ is defined,

$P_{I}\left(\pi_{2}\right)(u)$ is defined, aud

$P_{I}\left(\pi_{1}\right)(u)=P_{I}\left(\pi_{2}\right)(u)$, and

for each $\sigma \in \mathfrak{S}$ and cach $\pi \in \mathfrak{P}$,

$\langle\pi, \sigma\rangle \in \Lambda$

iff $P_{I}(\pi)(u)$ is defined, and $\sigma=S\left(P_{I}(\pi)(u)\right)$.

Proposition 10. For each interpretation $I$ and each object $u$ in $I$,

some unique morph abstracts $u$ under $I$.

I thus write of the abstraction of $u$ under $I$.

Definition 11. $u$ is a standard olject iff

$u$ is a quadruple $\langle\Delta, \mathrm{\Gamma}, \Lambda, \mathbf{E}\rangle$,

$\langle\Delta, l, \Lambda\rangle$ is a morph, and

$\mathrm{E}$ is an equivalence class under I'.

I write $\widetilde{U}$ for the set of standard objects, write

$\widetilde{S}$ for the total function from $\widetilde{U}$ to $\mathcal{G}$, where

for each $\sigma \in \mathfrak{S}$ and each $\langle\Delta, \mathrm{I}, \Lambda, \mathrm{E}\rangle \in \widetilde{U}$,

$\widetilde{S}\langle\Delta, \Gamma, \Lambda, \mathbf{E}\rangle=\sigma$

iff for some $\pi \in \mathrm{E}, \Lambda(\pi)=\sigma$,

and write $\tilde{A}$ for the total function from $\mathfrak{A}$ to the set of partial functions from $\widetilde{U}$ to $\widetilde{U}$, where

for each $\alpha \in \mathfrak{A}$, each $\langle\Delta, \Gamma, \Lambda, \Gamma\rangle \in \widetilde{U}$ and $\operatorname{each}\left\langle\Delta^{\prime}, \Gamma^{\prime}, \Lambda^{\prime}, \mathbf{F}^{\prime}\right\rangle \in \widetilde{U}$,

$\widetilde{\Lambda}(\alpha)\langle\Delta, \Gamma, \Lambda, F\rangle$ is defined, and

$\widetilde{A}(\alpha)\left\langle\Delta, \mathrm{l}^{\prime}, \Lambda, \mathrm{E}\right\rangle=\left\langle\Delta^{\prime}, \Gamma^{\prime}, \Lambda^{\prime}, \mathrm{l}_{\prime}^{\prime}\right\rangle$

iff $\langle\Delta, \mathbf{\Gamma}, \Lambda\rangle=\left\langle\Delta^{\prime}, \Gamma^{\prime}, \Lambda^{\prime}\right\rangle$, and

for some $\pi \in \mathrm{E}, \pi \alpha \in \mathbf{E}^{\prime}$

Lemma 12. $(\widetilde{U}, \widetilde{S}, \widetilde{A}\rangle$ is an interpretation.

I write $\widetilde{I}$ for $\langle\widetilde{U}, \widetilde{S}, \widetilde{A}\rangle$.

Lemma 13. For each $\langle\Delta, \Gamma, \Lambda, \mathrm{L}\rangle \in \tilde{U}$, ach $\left\langle\Delta^{\prime}, \Gamma^{\prime}, \Lambda^{\prime}, \mathrm{F}^{\prime}\right\rangle \in \widetilde{U}$ and each $\pi \in \mathfrak{P}$, $P(\pi)(\Delta, \mathrm{I}, \Lambda, \mathrm{F}\rangle)$ is defined, and $P_{\widetilde{I}}(\pi)\langle\Delta, \mathrm{I}, \Lambda, \mathrm{E}\rangle=\left\langle\Delta^{\prime}, \Gamma^{\prime}, \Lambda^{\prime}, \mathrm{E}^{\prime}\right\rangle$ iff $\left\langle\Delta, \mathbf{I}^{\prime}, \Lambda\right\rangle=\left\langle\Delta^{\prime}, \Gamma^{\prime}, \Lambda^{\prime}\right\rangle$, and

for some $\pi^{\prime} \in \mathbf{L}, \pi^{\prime} \pi \in \mathbf{E}^{\prime}$.

Proof. By induction on the length of $\pi$.

Lemma 14. For $\operatorname{each}\langle\Delta, I, \Lambda, E\rangle \in \widetilde{U}$,

if $\mathrm{E}$ is the equivalence class of the cmpty path under $\mathbf{I}$

then the abstraction of $\langle\Delta, \Gamma, \Lambda, \omega\rangle$ under $\tilde{I}$ is $\langle\Delta, \Gamma, \Lambda\rangle$.

Proposition 15. For each morph $M$,

for some interpretation $I$ and some object $u$ in $I$,

$M$ is the abstraction of $u$ under $I$.
Definition 16. $F$ approximates $M$ iff $F$ is a feature structure $\langle Q, q, \delta, \theta\rangle$, $M$ is a morph $\langle\Delta, \Gamma, \Lambda\rangle$, and

for each $\pi_{1} \in \mathfrak{P}$, each $\pi_{2} \in \mathfrak{P}$ and each $q^{\prime} \in Q$,

if $\pi_{1}$ runs to $q^{\prime}$ in $F$, and

$\pi_{2}$ runs to $q^{\prime}$ in $F$

then $\left\langle\pi_{1}, \pi_{2}\right\rangle \in \Gamma$, and

$$
\theta\left(q^{\prime}\right) \preceq \Lambda\left(\pi_{1}\right)
$$

$\Lambda$ feature structure approximates a morph iff the Moshier abstraction of the feature structure abstractly subsumes (see [CARPENTER 1992]) the morph.

Proposition 17. For each interpretation I, each object $u$ in $I$ and each feature structure F,

$F$ is true of $u$ under $I$

iff $F$ approximates the abstraction of $u$ under $l$.

Theorem 18. For each feature structure $F$, $F$ is satisfiable iff $F$ approximates some morph.

Proof. From propositions 15 and 17.

\section{RESOLVED FEATURE STRUCTURES}

'Though theorem 18 gives an interpretation free characterisation of a satisfiable feature structure, the characterisation still seems to admit of no eflective algorithm to decide if a feature structure is satisfiable. However, I use theorem 18 and resolved feature structures to yield a less general interpretation free characterisation of a satisfiable feature structure that admits of such an algorithm.

Definition 19. $R$ is a resolved fealure structure iff

$R$ is a foature structure $\langle Q, q, \delta, \rho\rangle$, $\rho$ is a total function from $Q$ to $\mathfrak{S}$, and for cach $\alpha \in \mathfrak{A}$ and each $q^{\prime} \in Q$,

if $\delta\left(q^{\prime}, \alpha\right)$ is defined

then $\mathfrak{F}\left(\rho\left(q^{\prime}\right), \alpha\right)$ is defined, and $\mathfrak{F}\left(\rho\left(q^{\prime}\right), \alpha\right) \preceq \rho\left(\delta\left(q^{\prime}, \alpha\right)\right)$.

Each resolved feature structure is a well-typed (see [CARPEN'TER 1992]) feature structure with output alphabet $\mathfrak{S}$.

Definition 20. $R$ is a resolvant of $F$ iff $R$ is a resolved feature structure $\langle Q, q, \delta, \rho\rangle$, $F$ is a feature structure $\langle Q, q, \delta, 0\rangle$, and for each $q^{\prime} \in Q, O\left(q^{\prime}\right) \preceq p\left(q^{\prime}\right)$.

Proposition 21. For each interpretation $I$, each object $u$ in $I$ and each feature structure $F$,

$F$ is true of $u$ under $I$

iff some resolvant of $F$ is true of $u$ under $I$. 
Definition 22. $\langle\mathfrak{Q}, \mathfrak{T}, \preceq, \mathfrak{G}, \mathfrak{A}, \mathfrak{F}\rangle$ is rational iff for each $\sigma \in \mathfrak{S}$ and each $\alpha \in \mathfrak{A}$,

if $\mathfrak{F}(\sigma, \alpha)$ is defined

then for some $\sigma^{\prime} \in \mathfrak{G}, \mathfrak{F}(\sigma, \alpha) \leq \sigma^{\prime}$.

Proposition 23. If $\langle\mathfrak{Q}, \mathfrak{T}, \preceq, \mathfrak{S}, \mathfrak{A}, \mathfrak{F}\rangle$ is rational then for each resolved feature structure $R, R$ is satisfiable.

Proof. Suppose that $R=\langle Q, q, \delta, \rho\rangle$ and $\beta$ is a bijection from ordinal $\zeta$ to $\mathcal{G}$. Let

$$
\begin{aligned}
& \Delta_{0}=\left\{\pi \mid \begin{array}{c}
\text { for some } q^{\prime} \in Q, \\
\pi \text { runs to } q^{\prime} \text { in } R
\end{array}\right\}, \\
& \Gamma_{0}=\left\{\begin{array}{l|l}
\left\langle\pi_{1}, \pi_{2}\right\rangle & \begin{array}{l}
\text { for some } q^{\prime} \in Q, \\
\pi_{1} \text { rums to } q^{\prime} \text { in } R, \text { and } \\
\pi_{2} \text { rums to } q^{\prime} \text { in } R
\end{array}
\end{array}\right\} \text {, } \\
& \text { and } \\
& \Lambda_{0}=\left\{\begin{array}{l|l}
\langle\pi, \sigma\rangle & \begin{array}{l}
\text { for some } q^{\prime} \in Q, \\
\pi \text { runs to } q^{\prime} \text { in } h, \text { and } \\
\sigma=\rho\left(q^{\prime}\right)
\end{array}
\end{array}\right\} .
\end{aligned}
$$

For each $n \in \mathrm{IN}$, let

$$
\begin{aligned}
& \Delta_{n+1}= \\
& \Delta_{n} \cup\left\{\begin{array}{l}
\pi \alpha \\
\begin{array}{l}
\alpha \in \mathfrak{A}, \\
\pi \in \Delta_{n}, \text { and } \\
\mathfrak{F}\left(\Lambda_{n}(\pi), \alpha\right) \text { is defined }
\end{array}
\end{array}\right\}, \\
& 1_{n+1}= \\
& \mathrm{I}_{n} \cup\left\{\begin{array}{l|l}
\left\langle\pi_{1} \alpha, \pi_{2} \alpha\right\rangle & \begin{array}{l}
\alpha \in \mathfrak{A}, \\
\pi_{1} \alpha \in \Delta_{n+1}, \\
\pi_{2} \alpha \in \Delta_{n+1}, \text { and } \\
\left\langle\pi_{1}, \pi_{2}\right\rangle \in \Gamma_{n},
\end{array}
\end{array}\right\} \text {, and } \\
& \Lambda_{n+1}= \\
& \Lambda_{n} \cup\left\{\begin{array}{l|l}
\langle\alpha, \beta(\xi)\rangle & \begin{array}{l}
\alpha \in \mathfrak{Q}, \\
\pi \in \Delta_{n}, \\
\pi \alpha \in \Delta_{n+1} \backslash \Delta_{n}, \text { and } \\
\xi \text { is the least ordinal } \\
\text { in } \zeta \text { such that, } \\
\Im\left(\Lambda_{n}(\pi), \alpha\right) \preceq \beta(\xi)
\end{array}
\end{array}\right\} .
\end{aligned}
$$

For each $n \in \mathbb{N},\left\langle\Delta_{n}, l_{n}, \Lambda_{n}\right\rangle$ is a semi-morph lict

$$
\begin{aligned}
& \Delta=\bigcup\left\{\Delta_{n} \mid n \in \mathbb{I N}\right\}, \\
& \Gamma=\bigcup\left\{\mathbf{I}_{n} \mid n \in \mathbb{I N}\right\}, \text { and } \\
& \Lambda=\bigcup\left\{\Lambda_{n} \mid n \in \mathbb{I N}\right\} .
\end{aligned}
$$

$\langle\Delta, \Gamma, \Lambda\rangle$ is a morph that $l$ approximates. By theorem $18, R$ is satisfiable.

Theorem 24. If $(\mathfrak{Q}, \mathfrak{T}, \preceq, \mathfrak{S}, \mathfrak{Q}, \mathfrak{Z})$ is rational then for each feature structure $F$,

$H^{\prime}$ is satisfiable iff $F^{\prime}$ has a resolvant.

Proof. From propositions 21 and 23.

\section{A SATISFIABILITY ALGORITHM}

In this section, I use theorem 24 to show how given a rational signature 1. hat moets reasonable computational conditions -.. to construct an effective algorithm to deciele if a reature structure is salisfiable.
Definition 25. $\langle\mathfrak{Q}, \mathfrak{T}, \preceq, \mathfrak{S}, \mathfrak{A}, \mathfrak{F}\rangle$ is computable if

$\mathfrak{Q}, \mathfrak{T}$ and $\mathfrak{A}$ are conntable, $\mathcal{E}$ is finite, for some effective function SUB,

for each $\tau_{1} \in \mathfrak{T}$ and each $\tau_{2} \in \mathfrak{T}$,

if $\tau_{1} \preceq \tau_{2}$

then $\operatorname{SuB}\left(\tau_{1}, \tau_{2}\right)=$ 'true'

otherwise $\operatorname{SUB}\left(\tau_{1}, \tau_{2}\right)=$ 'false', and

for some effective function APP,

for each $\tau \in \mathfrak{T}$ and each $\alpha \in \mathfrak{A}$,

if $\mathfrak{F}(\tau, \alpha)$ is defined

then $\operatorname{APP}(\tau, \alpha)=\mathfrak{F}(\tau, \alpha)$

otherwise $\operatorname{APP}(\tau, \alpha)=$ 'undefined'.

Proposition 26. If $\langle\mathfrak{Q}, \mathfrak{T}, \preceq, \mathfrak{G}, \mathfrak{A}, \mathfrak{F}\rangle$ is computable then for some effective function RES,

for each feature structure $F$,

$\operatorname{RES}\left(F^{\prime}\right)=$ a list of the resolvants of $F^{\prime}$

Proof. Since $(\mathfrak{Q}, \mathfrak{T}, \preceq, \mathfrak{G}, \mathfrak{U}, \mathfrak{F}\rangle$ is computable, for some effective function GEN,

for each linites $Q \subseteq \mathfrak{Q}$,

$\operatorname{GEN}(Q)=$ a list of the total functions from $Q$ to $\mathrm{S}$,

for some effective function TEST 1 ,

for each finite set $Q$, each finite partial

function $\delta$ from the Cartesian product of $Q$ and $\mathfrak{a}$ t.o $Q$, and oach total function $\theta$ from $Q$ to 5

if for each $\langle q, \alpha\rangle$ in the domain of $\delta$, $\mathfrak{F}(O(q), \alpha)$ is defined, and $\mathfrak{F}(\theta(q), \alpha) \preceq \theta(\delta(q, \alpha))$

1. hen $\operatorname{TEST}_{1}(\delta, 0)=$ 'true'

ollerwise $\operatorname{TEST}_{1}(\delta, \theta)=$ 'false',

and for some effective function TEST,

for each finite set $Q$, each total function $O_{1}$ from $Q$ lo $T$ and each total function $\theta_{2}$ from $Q$ lo $\mathbb{T}$,

if for cach $q \in Q, O_{1}(q) \preceq O_{2}(q)$

then $\operatorname{TEST}_{2}\left(\theta_{1}, \theta_{2}\right)=$ 'true'

otherwise $\operatorname{TEST}_{2}\left(\theta_{1}, \theta_{2}\right)=$ 'false'.

Construct, RES as follows:

for each feature structure $(Q, q, \delta, 0)$,

set $\Sigma_{\text {in }}=\operatorname{GEN}(Q)$ and $\Sigma_{\text {out }}=\langle$

while $\Sigma_{\mathrm{in}_{1}}=\left\langle\rho, \rho_{1}, \ldots, \rho_{i}\right\rangle$ is not empty

do sot $\Sigma_{\text {in }}=\left\langle\rho_{1}, \ldots, \rho_{i}\right\rangle$

if $\operatorname{TEST}_{1}(\delta, \rho)=$ 'truc', $\operatorname{TEST}_{2}(\theta, \rho)=$ 'true', and $\Sigma_{\text {out }}=\left\langle\rho_{1}^{\prime}, \ldots, \rho_{j}^{\prime}\right\rangle$ then set $\Sigma_{\text {out }}=\left\langle\rho, \rho_{1}^{\prime}, \ldots, \rho_{j}^{\prime}\right\rangle$

if $\Sigma_{\text {out }}=\left\langle\rho_{1}, \ldots, \rho_{n}\right\rangle$

then output $\left\langle\left\langle Q, q, \delta, \rho_{1}\right\rangle, \ldots,\left\langle Q, q, \delta, \rho_{n}\right\rangle\right\rangle$.

RES is an effective algorithm, and

for each foature structure $l$,

$\operatorname{RES}\left(F^{\prime}\right)=$ a list of the resolvants of $r$. 
Theorem 27. If $\langle\mathfrak{Q}, \mathfrak{T}, \preceq, \mathfrak{S}, \mathfrak{A}, \mathfrak{F}\rangle$ is rational and computable then for some offective function SAT,

for each feature structure $r$, if $F$ is satisfiable

then $\operatorname{SAT}(F)=$ 'true

otherwise $\operatorname{SaT}(F)=$ 'false'.

Proof. From theorem 24 and proposition 26.

Gerdemann and Götz's Troll system (see [GöTz 1993], [GFrdimann and King 1994] and [Gerdemann (FC)]) employs an efficient refinement of RES to test the satisfiability of feature structures. In fact, 'Troll represents each feature structure as a disjunction of the resolvants of the feature structure. Loosely speaking, the resolvants of a feature structure have the same underlying firite state antomaton as the feature structure, and differ only in their output function. Troll exploits this property to represent each feature structure as a finite state automaton and a set of output functions. The Troll unifier is closed on these representations. 'Thus, though RES is computationally expensive, Troll uses RES only during compilation, never during run time.

\section{References}

[Carpenter 1992] Robert Carpenter The logic of typed feature structures. Cambridge tracts in theoretical computer science 32. Cambridge University Press, Cambridge, England. 1992.

[Gmrdemann (FC)] Dale Gerdemann. Troll: type resolution system, uscr's guide. Sonderforschungsbereich 340 technical report. Eberhard-Karls-Universität, Tübingen, Germany. Forthcoming.

[Gerdemann anis King (1994)] Dale Gerdemann and Paul John King. The correct and efficient implementation of appropriateness specifications for typed feature structures. In these procedings.

[GöTz 1993] Thilo Götz. A normal form for typed feature structures. Master's thesis. Fherhard-Karls-Universität, Tübingen, Germany. 1993.

[KIng 1989] Paul Joln King. A logical formalism for head-driven phrase structure grammar. Doctoral thesis. The University of Manchester, Manchester, England, 1989.

[Moore 1956] E. F. Moore. 'Gedanken experiments on secquential machines'. In $A$ utomata Studies. Princeton University P'ress, Princeton, New Jersey, USA. 1956.
[Moshifr 1988] Michael Andrew Moshier. Extensions to unification grammar for the description of programming languages. Doctoral thesis. The University of Michigan, Ann Arbor, Michigan, USA. 1988. 